\title{
The design and construction of a spectrometer to efficiently identify nuclear levels
}

\author{
K. Furutaka ${ }^{1, \text { a }}$, M. Oshima ${ }^{1}$, A. Kimura ${ }^{1}$, Y. Toh ${ }^{1}$, M. Koizumi ${ }^{1}$, T. Kin ${ }^{1}$, and J. Goto ${ }^{2}$ \\ Japan Atomic Energy Agency, 2-4 Shirakata-Shirane, Tokai-mura, Ibaraki 319-1195, Japan \\ 2 Niigata University, 1-757 Asahi-machi-dori, Niigata-shi, Niigata 951-8510, Japan
}

\begin{abstract}
A simulation study was performed to choose an optimal configuration of a gamma-ray spectrometer consisting of $\mathrm{Ge}$ and $\mathrm{BGO}$ detectors, which facilitates the identification of prompt gamma rays to ground states emitted following capture reactions of neutrons in the thermal energy region and determination of the reaction cross sections.
\end{abstract}

\section{Introduction}

The measurement of prompt gamma rays is a powerful tool to determine nuclear data such as capture cross sections of neutrons at, e.g., thermal energy. In particular, it is the only method to precisely determine neutron capture cross sections of nuclides which produce stable reaction products and therefore activation methods can not be applied; among them are Long Lived Fission Products (LLFP) such as ${ }^{79} \mathrm{Se}\left(T_{1 / 2}=\right.$ $\left.1.13 \times 10^{6} \mathrm{y}\right),{ }^{93} \mathrm{Zr}\left(1.53 \times 10^{6} \mathrm{y}\right)$, and ${ }^{107} \mathrm{Pd}\left(6.5 \times 10^{6} \mathrm{y}\right)$ (all the quoted half-lives are from ref. [1]).

There are at least three ways to determine neutron capture cross sections by measuring prompt gamma rays: (i) $\sum I_{\gamma}$ (primary), (ii) $\sum I_{\gamma}$ (to ground state), and (iii) $\sum E_{\gamma} I_{\gamma} / S_{n}$, where $I_{\gamma}$ stands for the intensity of a gamma ray, $E_{\gamma}$ for the energy, and $S_{n}$ for the neutron separation energy of the reaction product. The first two methods require the construction of a decay scheme and the identification of primary the last method, the construction of level schemes is not required, but it's essential to detect all the prompt gamma rays emitted following the capture reaction. In a contribution to the previous conference, one of the present authors has reported that in the ${ }^{99} \mathrm{Tc}$ (thermal $n, \gamma$ ) case, whose cross section can be determined both from yields of prompt gamma rays and in an activation method, the relation (ii) yielded about $96 \%$ of the cross section obtained in an activation method, whereas the relations (i) and (iii) amounted to significantly smaller values [2]; the cross section obtained through the relation (iii) is about $1 / 3$ of that of (ii) case, reaction suggest that the only feasible way would be from intensities of gamma transitions to ground states to determine capture cross sections of FP nuclides for thermal neutrons through prompt gamma-ray measurements.

To accurately determine the cross sections from intensities of ground-state transitions, it is essential to identify as many ground-state transitions and therefore nuclear levels as possible. One of the features of capture reactions of neutrons in the thermal energy region is that capturing states are relatively well defined in energy. Also, the neutrons bring a small amount of angular momentum and the multiplicity of prompt gamma rays is believed to be small. Therefore, it seems not so

${ }^{a}$ Presenting author, e-mail: furutaka.kazuyoshi@jaea.go.jp difficult to detect all gamma rays emitted following a single capture event, between the capture state and ground state. In such an event, the sum of energies of the observed gamma rays is equal to $S_{n}$. Hereafter in this paper such events will be called " $S_{n}$-energy events". In $S_{n}$-energy events, all cascading gamma rays from a capture state to a ground state are detected, and peaks in the observed gamma-ray spectra indicate differences in energy of the levels involved in cascades. Thus, such events greatly help identifying the levels below capture states. If one can efficiently collect $S_{n}$-energy events with good peak-tototal ratios using a gamma-ray spectrometer, such a system becomes a powerful tool for the identification of nuclear levels as well as ground-state transitions.

In order to facilitate the identification of nuclear levels and ground-state transitions, the present authors have decided to construct a gamma-ray spectrometer that is suited to observe $S_{n}$-energy events efficiently with adequate peak-to-total ratios for capture gamma rays, at an experimental station on one of the cold neutron beam lines of the JRR-3 research reactor, using germanium detectors detectors presently available to the authors and $\mathrm{BGO}\left(\mathrm{Bi}_{4} \mathrm{Ge}_{3} \mathrm{O}_{12}\right)$ scintillation detectors. To determine an optimal detector configuration for the spectrometer, the present authors have performed a simulation study, which will be described in the following.

\section{Details of the simulation}

\subsection{Sources of gamma rays and values characterizing performance of configurations}

The capture reaction of thermal neutrons by ${ }^{14} \mathrm{~N}$, ${ }^{14} \mathrm{~N}$ (thermal $\left.n, \gamma\right){ }^{15} \mathrm{~N}$ was chosen for the source of prompt gamma rays in the simulation; the reaction is safely said to be the most thoroughly studied and the decay scheme and intensities of the gamma rays have been well established $[3,4]$.

For the evaluation of the performance of each detector configuration, the following values were chosen:

1. The detection efficiency of $S_{n}$-energy events in the ${ }^{14} \mathrm{~N}($ thermal $n, \gamma){ }^{15} \mathrm{~N}$ reaction, i.e., events in which $\sum E_{\gamma}=$ $S_{n}=10,833 \mathrm{keV}$, 
2. The signal-to-noise $(S / N)$ ratio of 5269-keV gamma ray in $S_{n}$-energy events, which is a medium-energy and the most intense gamma ray in ${ }^{15} \mathrm{~N}$ (the signal and noise were defined as net peak area and square root of background area below the gamma-ray peak, respectively),

3. The detection efficiency of the most intense gamma cascade between the capture state and the ground state in ${ }^{15} \mathrm{~N}$, i.e., triple cascading gamma rays $(3678 \mathrm{keV} \rightarrow 1885 \mathrm{keV}$ $\rightarrow 5269 \mathrm{keV}$ ).

\subsection{Detector configuration and the simulation}

Germanium detectors which can be used for the spectrometer were:

- up to 14 coaxial-type detectors with detection efficiencies ranging from $15 \%$ up to $90 \%$,

- 8 Clover-type detectors (7 backcatcher-type and 1 standard), each containing 4 crystals (corresponding to about $120 \%$ detection efficiency), and

- 2 detectors with hammer-head shaped endcaps which contain either two $90 \%$ crystals (coaxial) or one $150 \%$ crystal (bullet shape with a non-coaxial hole for an electrode).

A dozen of possible detector configurations were designed using portions of the available detectors, some of which with higher performance are depicted in figure 1 .

The following objects were taken into consideration:

- Germanium detectors, and

- neutron beam guide tube.

Supporting frames for the detectors as well as the beam guide tube were not taken into account.

Only Ge crystals and their surrounding endcaps were considered. Detailed geometrical shapes of germanium crystals were input, whereas simplified shapes were used for their endcaps. Materials behind contact pins were not taken into consideration.

The neutron guide tube is made of LiF filled with the air whose inner cross section is rectangular $(40 \mathrm{~mm}$ in width and $70 \mathrm{~mm}$ in height). The thickness of the $\mathrm{LiF}$ is $20 \mathrm{~mm}$, and it is further shielded by $50 \mathrm{~mm}^{\mathrm{t}}$ lead. Within $\pm 13 \mathrm{~cm}$ from its neutron irradiation position, the thickness of the $\mathrm{LiF}$ is reduced to $10 \mathrm{~mm}$ and the lead shield is removed so that attenuation of gamma rays is reduced and detectors can be set as close to the irradiation position as possible. Only a part of the guide tube within $\pm(50+13) \mathrm{cm}$ from the irradiation position was taken into account (see fig. 1(e)).

The simulation was done using the GEANT4 toolkit [5, 6]. A total of $10^{7}$ events were simulated for each detector configuration. In each event a ${ }^{15} \mathrm{~N}$ nucleus in its neutron capture state at $10,833 \mathrm{keV}$ is placed at the irradiation position of the beam line and gamma rays emitted through its decay is followed using the toolkit, and energy deposited in each detector crystal was recorded on event-by-event basis. The neutron capture state in ${ }^{15} \mathrm{~N}$ is not in "the adopted levels and gammas" of ${ }^{15} \mathrm{~N}$ in the ENSDF [7] and therefore it is not included in the standard distribution of the data files for GEANT4. So the radiative decay data of ${ }^{15} \mathrm{~N}$ was modified to incorporate the capture state.

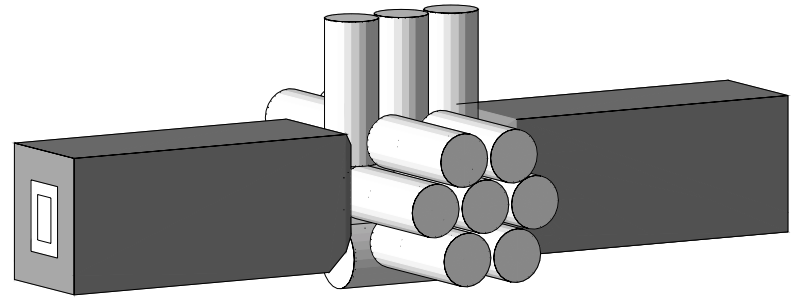

(a) Configuration "A": 17 coaxial Ge's above and on both sides of the beam guide, and a hammer-head shaped Ge below the beam guide.

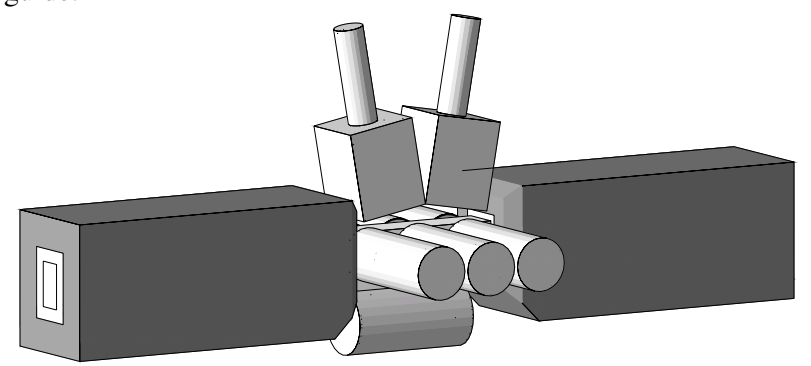

(b) Configuration "B": 6 coaxial Ge's on both sides of the beam guide, and a hammer-head shaped Ge below and two clovershaped Ge's above the beam guide.

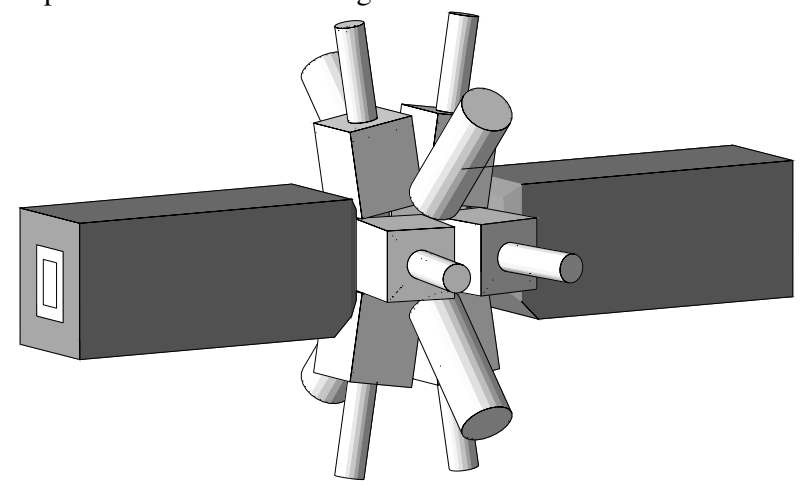

(c) Configuration "C": 8 clover-shaped Ge's on both sides of and above/below the beam guide, and 4 coaxial Ge's mounted slant.

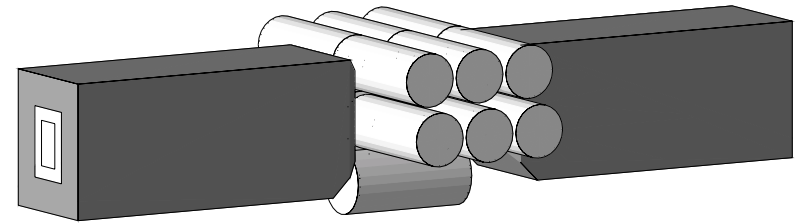

(d) Configuration "D": 12 coaxial Ge's set on both sides as well as above the beam guide, and a hammer-head shaped Ge mounted below the beam guide.

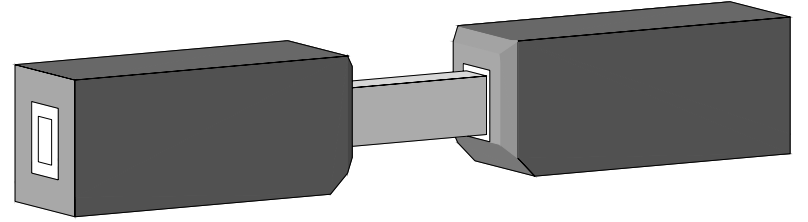

(e) Bare neutron beam guide within $\pm 63 \mathrm{~cm}$ from the irradiation position along the neutron beam.

Fig. 1. A part of detector configurations designed, which attained higher performance in the simulation, and the bare neutron guide tube. All the coaxial Ge's are with $70 \%$ relative efficiency. Only the materials taken into account in the simulation are depicted. 


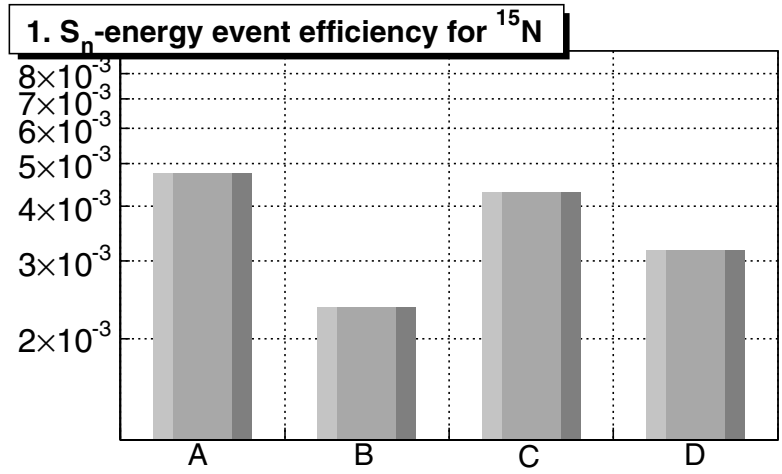

\section{S/N of $5269-k e V \gamma$ in ${ }^{15} \mathrm{~N}$}

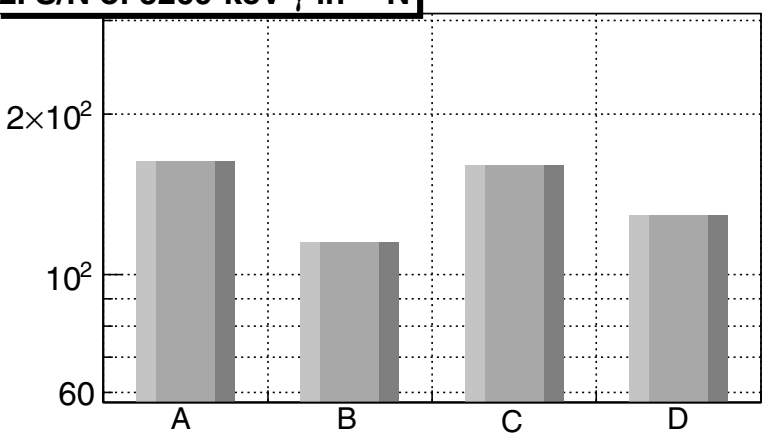

\section{Coin.Eff. of $3 \gamma s$ in ${ }^{15} \mathrm{~N}$}

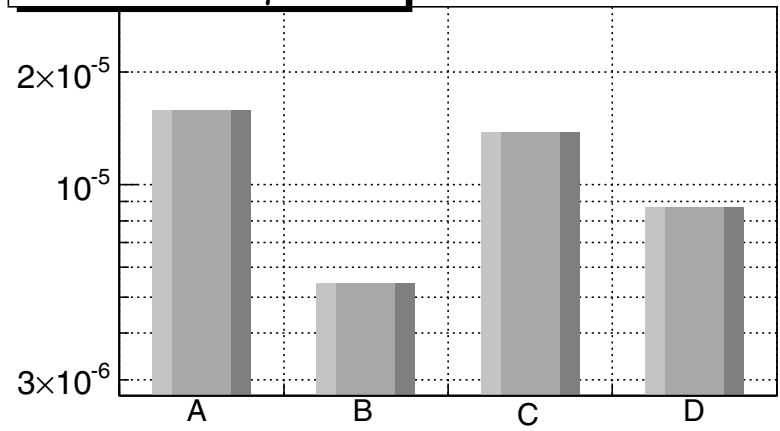

Fig. 2. Comparison of values characterizing the detector configurations shown in the figure 1 . Labels on the abscissas indicate the detector configurations.

\section{Results and discussion}

The obtained data were analyzed off-line, and values described in the section 2.1 were deduced to evaluate each detector configuration. In the analysis, the finite energy resolution of $\mathrm{Ge}$ detectors is taken into account. A Gaussian shape was assumed for the broadening, and width of the Gaussian was assumed to be proportional to $\sqrt{E_{\gamma}}$ and reproduces a typical value $(\sim 2.54 \mathrm{keV}$ at $1,332 \mathrm{keV})$ obtained by clovertype Ge detectors. Chance coincidence due to finite recording width of an event was also taking into account by assuming $100 \mathrm{k}$ events per second and $1 \mu \mathrm{s}$ coincidence width. For all detector configurations designed, the values indicative of their performance were calculated. Among all the setups, the ones shown in figures 1(a) 1(d) attained higher performance. For these four configurations, the obtained values are compared in figure 2 .
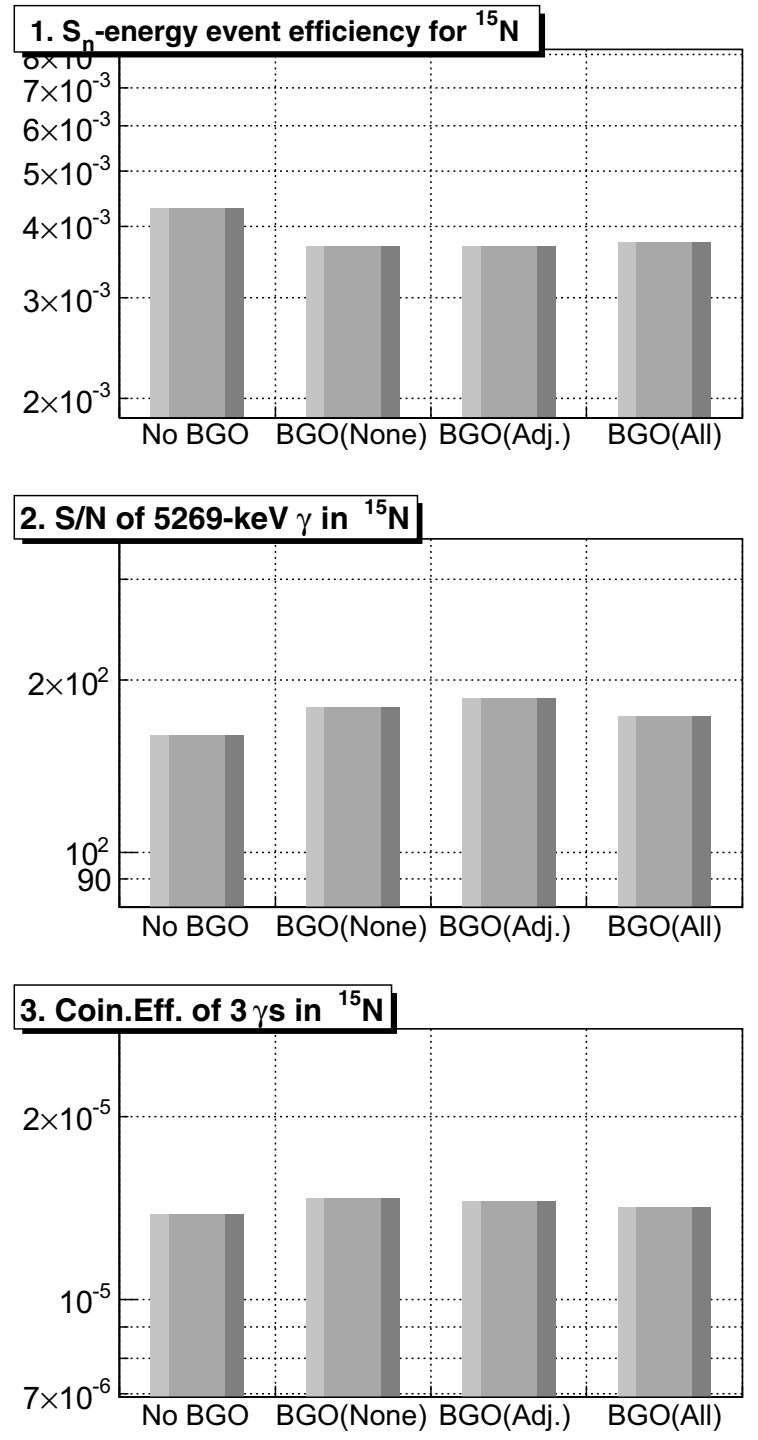

Fig. 3. Comparison of values characterizing the detector configuration "C" with and without BGO escape suppressors. "No BGO": The same results as in figure 2. "BGO(None)": The Ge detectors surrounded by BGO detectors, but none of the BGO detectors are operated as escape suppressors. "BGO(Adj.)": The Ge detectors surrounded by BGO detectors, and the BGO detectors only adjacent to a Ge detector are operated as the escape suppressors of the Ge. "BGO(All)": The Ge detectors surrounded by BGO detectors, and events are suppressed if any of the BGO detectors are hit.

Looking at the figure, it is apparent that all four values show the same trend. Also apparent is that the configurations "A" and "C" are the two with the highest and almost the same performance, so the choice was whether to choose the "A" or "C".

As the primary purpose of this spectrometer is to detect all the cascading gamma rays between the capture states and ground states with a reasonable efficiency and to facilitate identification of nuclear levels, it is essential to observe gamma rays from low energy up to $S_{n}$ with good response, i.e., good peak-to-total ratios; higher $S_{n}$-energy event efficiency is one of the required conditions. To achieve good response, it is 


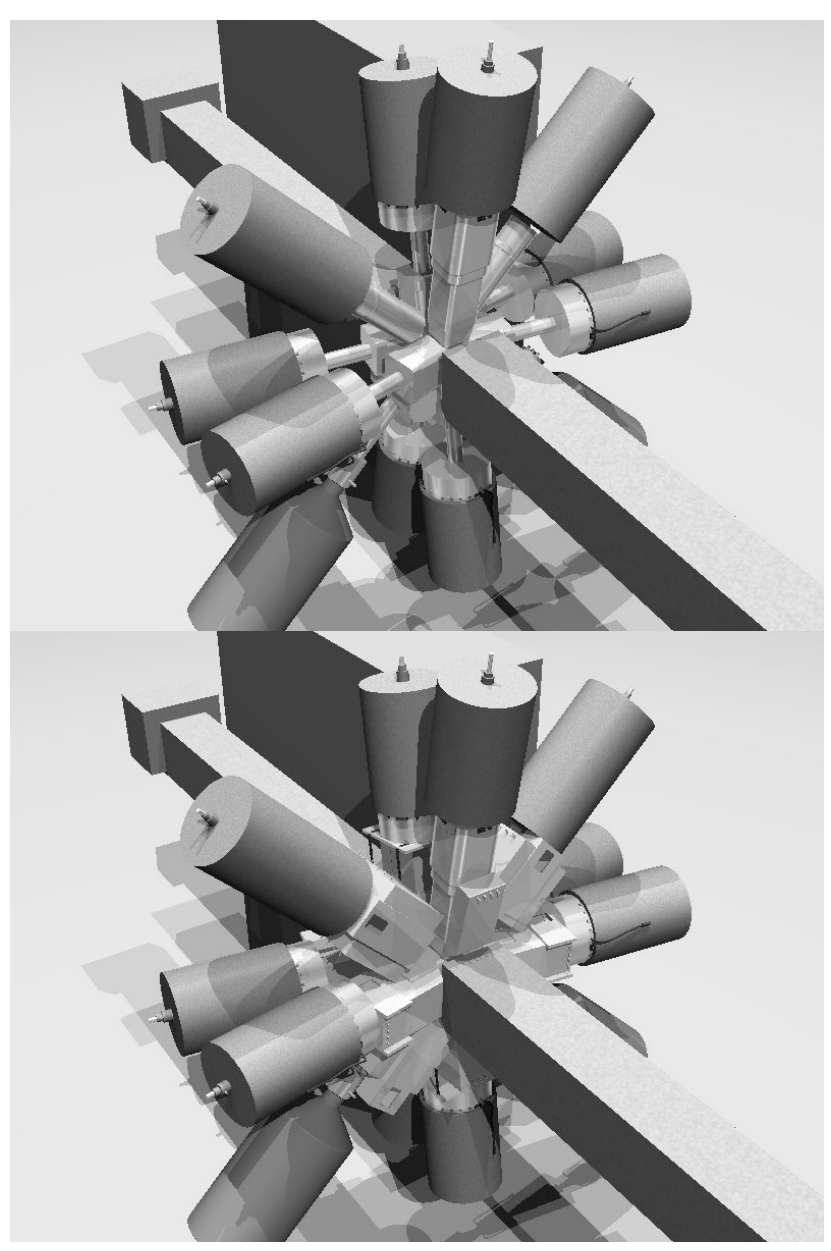

Fig. 4. The detector configuration chosen for the spectrometer, with (lower) and without (upper) the BGO detectors.

inevitable to reduce cross-talk between detectors. Considering this, a closely packed geometry of coaxial Ge detectors in the "A" configuration is disadvantageous for reducing cross-talk and the configuration " $\mathrm{C}$ " is more suited because there are spaces between $\mathrm{Ge}$ detectors into which a shielding material like lead or an active shield like BGO detectors can be inserted. So the present authors have chosen the configuration " $\mathrm{C}$ " for the basic setup of the spectrometer.

To see the effect of BGO detectors, the same simulation and analysis were performed for the configuration " $\mathrm{C}$ " with BGO detectors attached around the Ge detectors. The obtained results figure 3. From the figure one see that the mere insertion of the BGO detectors ("BGO(None)") reduced efficiency of $S_{n}$-energy events by about $14 \%$. This implies that about $14 \%$ of the $S_{n}$-energy events observed using the detectors in the bare "C" configuration includes contribution from cross-talk events in which part of a gamma ray was absorbed in a $\mathrm{Ge}$ detector and the remaining in the others. On the other hand, the insertion of the BGO detectors improve both $S / N$ of the 5269-keV gamma ray and the triple coincidence efficiency, which were resulted from reducing cross-talk and therefore sum-coincidence. These results show that the use of an active shield like BGO is advantageous.

To further improve the performance, two coaxial Ge detectors in the configuration can be replaced by the two hammerhead shaped ones. And finally, the configuration shown in figure 4 has been chosen for construction.

\section{Summary}

In order to facilitate the identification of nuclear levels and ground-state transitions, a simulation study was performed to choose an optimal design of a spectrometer which has a good efficiency for $S_{n}$-energy events and sufficient $S / N$ ratios for capture gamma rays. Based on the results of the simulation a configuration was chosen for the spectrometer, which comprises 8 clover-type Ge's, 2 coaxial Ge's, 2 hammer-head shaped Ge's, and surrounding BGO detectors. The spectrometer is now under construction.

\section{References}

1. R. Firestone, Table of isotopes (1998 Update with CD-ROM), 8th edn. (John Wiley \& Sons, Inc., New York, 1998).

2. K. Furutaka, H. Harada, S. Raman, Determination of the ${ }^{99}$ Tc (thermal $\left.n, \gamma\right){ }^{100}$ Tc Reaction Cross Section from the Prompt $\gamma$-Ray Yields, in International Conference On Nuclear Data For Science And TechnologY, edited by R.C. Haight, M.B. Chadwick, T. Kawano, P. Talou (American Institute of Physics, Melville, New York), Santa Fe, New Mexico, 2005), AIP Conf. Proc. 769, pp. 1454-1457.

3. E.T. Jurney, J.W. Starner, J.E. Lynn, S. Raman, Phys. Rev. C 56, 118 (1997).

4. S. Raman, C. Yonezawa, H. Matsue, H. Iimura, N. Shinohara, Nucl. Instrum. Meth. Phys. Res. A 454, 389 (2000).

5. S. Agostinelli, J. Allison, K. Amako, J. Apostolakis, H. Araujo, P. Arce, M. Asai, D. Axen, S. Banerjee, G. Barrand et al., Nucl. Instrum. Meth. Phys. Res. A 506, 250 (2003).

6. J. Allison, K. Amako, J. Apostolakis, H. Araujo, P. Arce Dubois, M. Asai, G. Barrand, R. Capra, S. Chauvie, R. Chytracek et al., IEEE Trans. Nucl. Sci. 53, 270 (2006).

7. http://www.nndc.bnl.gov/ensdf/ 